\title{
Postoperative Necrotizing Fasciitis of the Thorax in Cardiac Surgery
}

\author{
José Dario Frota Filho, Carlos Drews, Paulo Leães, Celso Blacher, Francisco Tostes, Nilton Delatorre, \\ Mariana Vieira, Eraldo Lúcio, Wagner Pereira, Marcela Sales, Roberto Lobo, Ralf Stuermer, \\ Nicasio Tanaka, Luis Portugal, Fernando Lucchese
}

Porto Alegre, RS - Brazil

Necrotizing fasciitis is a rare soft tissue infection and a life-threatening emergency, often fatal. Its incidence and management are described plentifully in the medical literature regarding the most common anatomical sites involved like the abdomen, lower and upper limbs, and perineum. However, available data and case reports of chest wall necrotizing fasciitis after thoracic procedures are scarce, mainly after major cardiac operations. We report and discuss a case of necrotizing fasciitis of the chest wall occurring in the immediate postoperative period of a cardiac procedure, and include a brief review of the concepts, pathophysiology, and treatment reported in the medical literature. We emphasize the need for early diagnosis and urgent and effective surgical debridement. Ofimportance is the fact that we have not found any references in the literature to cases similar or equal to the one we describe here, which occurred in the postoperative period of a cardiac procedure.

Necrotizing fasciitis of the chest wall is a rare disease that usually takes place as a complication of surgical procedures in the cervical region, spreading downward to the anterior chest wall, pericardium, and even the deep mediastinal structures ${ }^{1}$. Reports in the medical literature are very plentiful regarding necrotizing fasciitis occurring in other topographic body areas but otherwise scarce when focusing primarily on the thoracic incidence and management. Although the general therapeutic strategies used in necrotizing fasciitis are also applied to thoracic fasciitis, some special features exist that should be emphasized when dealing with thoracic necrotizing infections, beyond the unique postoperative issues involving either an intra- or extra-cardiac im-

Hospital São Francisco da Santa Casa de Porto Alegre

Mailling address: José Dario Frota Filho - Rua Freitas Cabral, 305/602 - 90690130 - Porto Alegre, RS planted prosthesis. The differential diagnosis between necrotizing fasciitis and non-necrotizing soft tissue infections should be a priority, as they present different prognostic and therapeutic implications ${ }^{2}$. In this brief communication, we focus on a literature review regarding concepts, pathophysiology, and treatment of necrotizing fasciitis and report and discuss a rare case of anterior chest wall necrotizing fasciitis as a complication of cardiac surgery. To our knowledge, no such cases have been reported in the medical literature.

\section{Case Report}

The patient is a 38-year-old male with Marfan's Syndrome, who presented with a huge aneurysm of the ascending aorta $(8 \mathrm{~cm}$ in diameter), severe aortic valve regurgitation, and severe left ventricular dysfunction. His previous medical history included septic shock as a complication in the postoperative period of an orthopedic procedure to the left ankle due to a traumatic fracture. At present, the surgical procedure was an aortoplasty with a composite Dacron and bovine pericardium graft (Labcor Laboratórios Belo Horizonte - Brazil), in which a double leaflet mechanical aortic prosthesis was attached Carbomedics Inc., USA, and coronary ostia reimplantation was performed. The immediate evolution included persistent fever and inflammatory signs at the upper and lower incision limits, with a yellowish discharge. Computerized tomography of the thorax and mediastinum showed a viscid pus-like collection around the aortic graft suggestive of mediastinitis. The sternum was stable. Incision and drainage of 2 incisional abscesses were performed. The infectious process spread through the neighboring tissues with formation of small abscesses, frankly purulent discharge, cellulites, and skin necrosis (Fig. 1). The first wide debridement, with resection of all apparently necrotic tissues, was performed, but soon we realized that it was ineffective (Fig. 2). Septic shock and local crepitus were established, and the diagnosis of necrotizing fasciitis of 


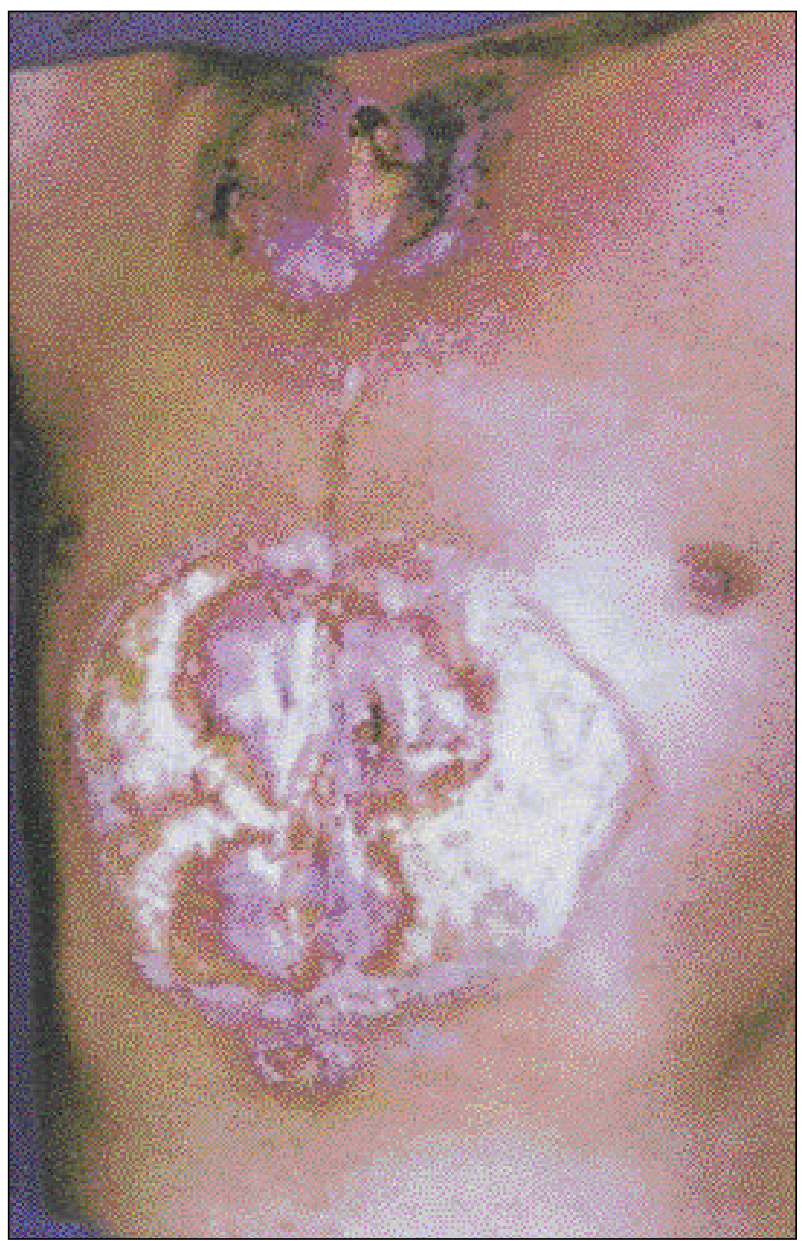

Fig. 1 - Soft tissue necrosis of the upper and lower thirds of the wound on the $8^{\text {th }}$ PO day.

the anterior chest wall was made. Broad-spectrum antibiotics (an association of vancomycin and cefazolin, and imipenem later on) were started together with fluid resuscitation and general cardiorespiratory supportive care. Aggressive surgical debridements were performed trying to reach normal bleeding-and healthy edges of the soft tissues (Fig. 3). We decided not to redo mediastinal exploration due to the sternal stability and lack of other local signs of mediastinitis. The necrotic fascia was easily detached from the muscular bed by blunt dissection. The progression of the necrotizing fasciitis kept on, and it was necessary to surgically remove affected areas on a daily basis, under general anesthesia, up to the point were the necrotizing fasciitis was completely controlled. No topical antibiotic or other agents were used as a routine to ease the identification of compromised tissues at the time of debridement. On the $14^{\text {th }}$ postoperative day, the patient was still presenting systemic signs of sepsis with loss of renal function. From the third week on, the infection was completely controlled, the multiorgan systemic response was almost normal, and exuberating granulation tissue existed (Fig. 4). Dressings were then applied at bedside with no need of general anesthesia. The plastic and reconstructive team was contacted, and the chest wall scar was repaired with skin flaps from the thighs, on the $51^{\text {st }}$

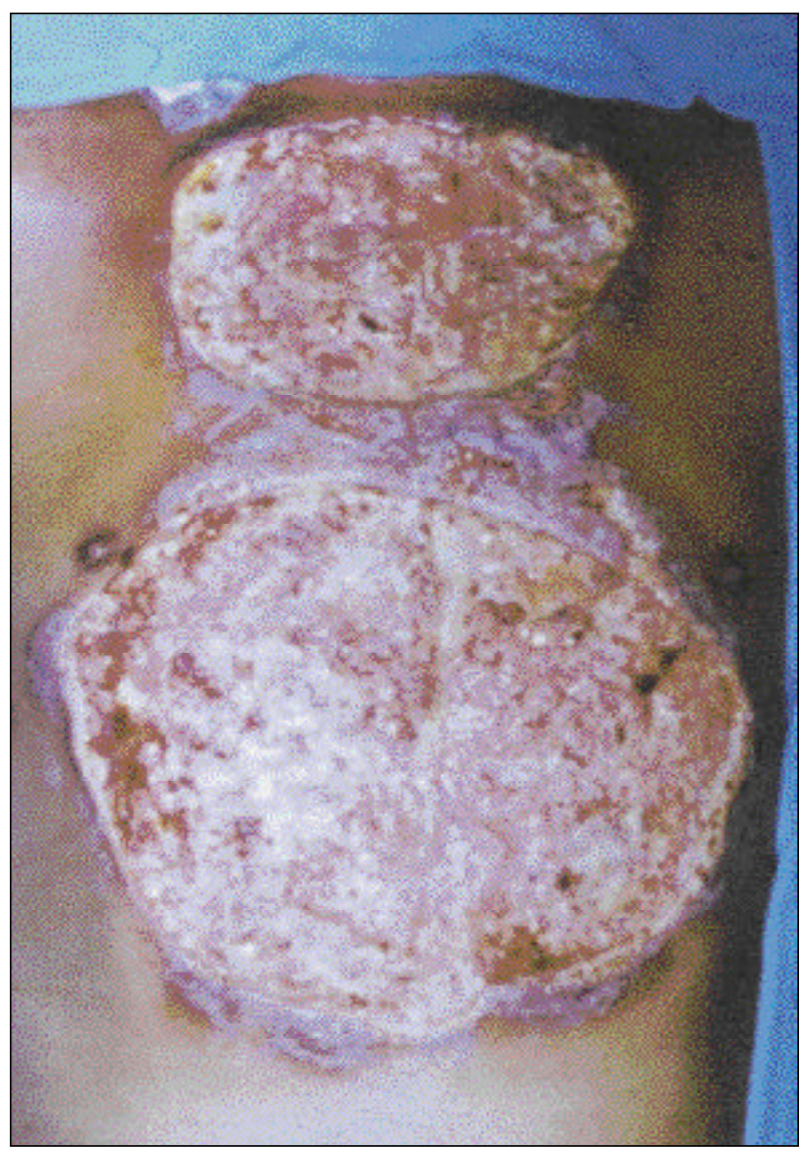

Fig. 2 - The soft tissue necrosis was still in progress after the initial debridement.

postoperative day (Fig. 5). The viscid pus-like collection first seen on computerized tomography scan had already completely subsided, and the sternum was still stable. The patient completely recovered and was discharged. Of utmost importance in the reported case is the fact that none of the cultures from various materials were positive (pus, blood, fragments of tissues, and temporary pacemaker wires).

\section{Discussion}

Necrotizing fasciitis was first mentioned by Hippocrates around the $5^{\text {th }}$ century A.D. as a complication of erysipela. Later on, the first case was reported by Meleney in $1924^{3}$. Quite different from erysipela, necrotizing fasciitis is an uncommon but highly lethal soft tissue infection caused by virulent organisms and their necrotoxins, mainly Group A beta-hemolytic Streptococcus ${ }^{4}$. Extensive fascial necrosis, including also the skin and subcutaneous tissue, systemic toxicity, septic shock, multiorgan failure and death, unless promptly diagnosed and treated, are characteristic of necrotizing fasciitis. The medical literature widely describes necrotizing fasciitis taking place in various anatomical regions. However, references about thoracic necrotizing fasciitis occurring primarily as a complication of thoracic procedures are scarce. Only 9 cases had been reported on up to 


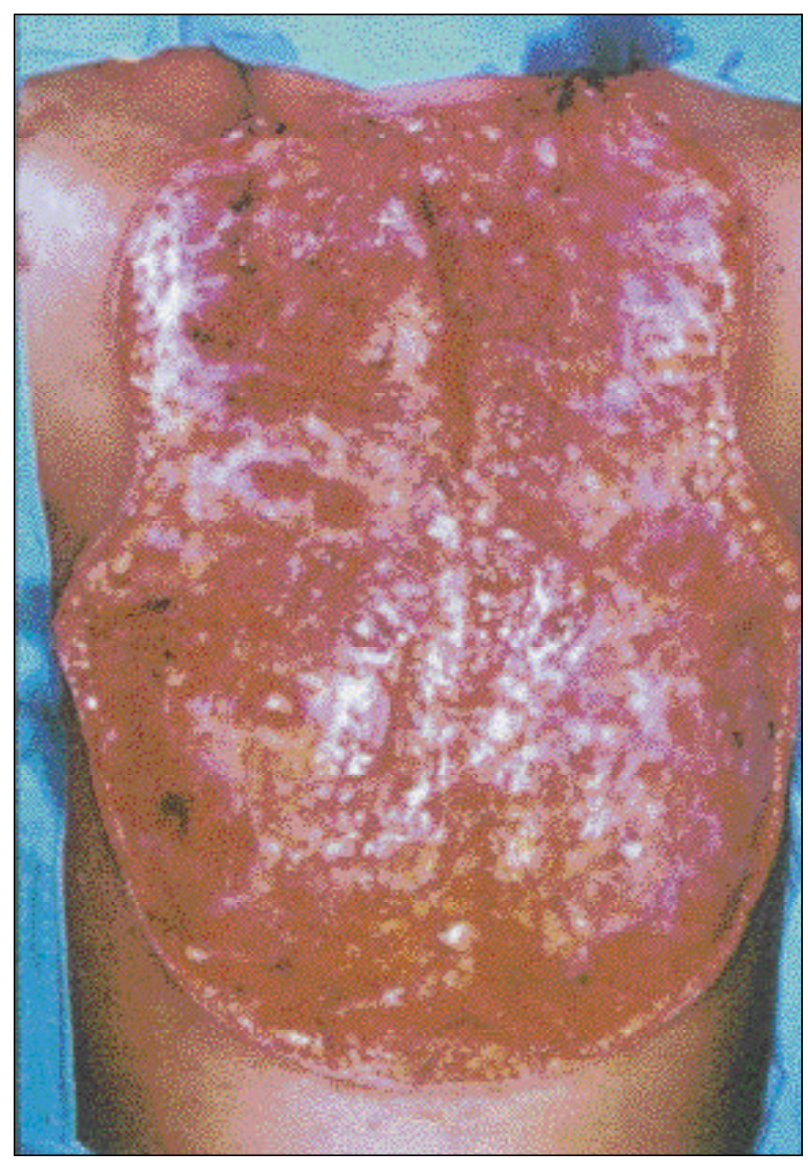

Fig. 3 - Aggressive surgical debridement extending up to the anterior axillary lines, the base of the neck and the epigastric region. Infection was already controlled.

1997, including 4 cases after tube thoracostomy for empyema, 3 cases after gastroesophageal surgery (repair of spontaneous esophageal rupture, repair of gastritis and esophagectomy for cancer), 1 case after pneumonectomy, and 1 case after thoracic trauma ${ }^{5}$. Probably some more cases exist that unfortunately have not been reported up to the present. Of great importance is the fact that we have not found any literature reference about chest wall necrotizing fasciitis as a complication of an intracardiac procedure.

Soft tissue necrotizing infections are more likely to occur in immunologically compromised individuals, chiefly in diabetics. Thirty-three percent of all postoperative suppurative complications of thoracic surgery, in the main due to deficiencies in the immunologic state of patients, were reported in children with inherited syndromes of the connective tissue. The most serious immunologic disturbances were observed in children with Marfan's syndrome, due to inadequate phagocytosis of the neutrophils and monocytes and a decreased number of T, active T, and B linfocytes ${ }^{6}$.

In very rare occasions, only one organism is responsible for the devastating fascial necrosis and systemic toxicity seen in necrotizing fasciitis (eg, necrotizing fasciitis caused by Streptococcus pyogenes). In the vast majority of cases, the synergistic action of aerobic and anaerobic organisms is responsible for a polymicrobial infection. Facultati-

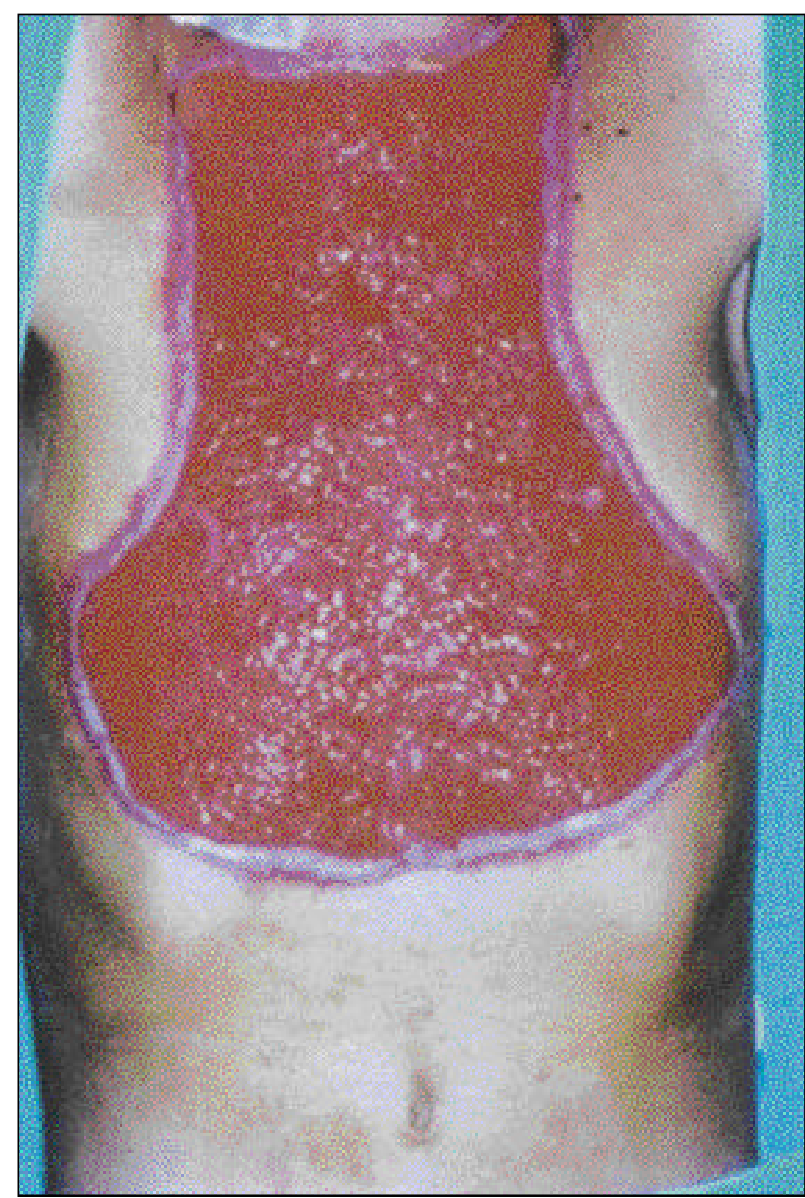

Fig.4 - Secondary healing of the margins in order to decrease the size of the recipient area.

ve organisms reduce the oxidation-reduction potential of the wound and promote favorable conditions for the growth of anaerobes. These last, in turn, impair the host phagocyte function and hence facilitate the growth of aerobes. Several bacteria, such as Bacteroides fragilis, produce beta-lactamase enzyme, which lessens antibiotic action. The number and kind of bacteria is related to the region affected ${ }^{7,8}$. The most frequently identified organisms include aerobic and anaerobic streptococcus, coagulantpositive and coagulant-negative staphylococcus, facultative and aerobic Gram-negative rods, bacteroid species and Clostridium ${ }^{5}$.

The necrotic alterations are directly related to the infectious process, with activation of the coagulation system, resulting in local thrombosis and infarction, with the aid of bacterial heparinase production. Tissue ischemia and anoxia, together with edema, promote increased pressures within the soft tissues and further impair the blood supply.

The diagnosis is essentially made on clinical grounds ${ }^{9}$ and requires an attitude of high suspicion because the early soft tissue alterations are unspecific. The external appearance of the skin wound may have no correlation with the magnitude of the deep fascial necrosis, being a factor that contributes to a diagnostic delay. The characteristic signs 


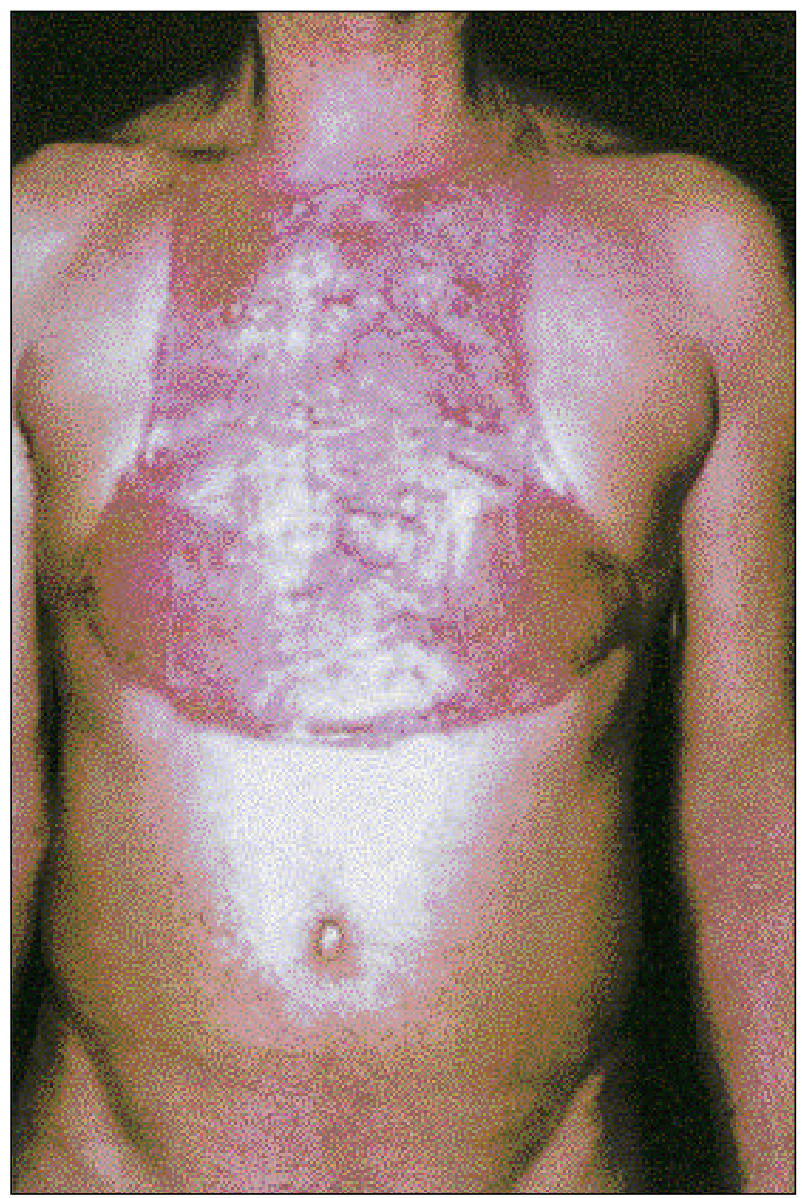

Fig. 5 - At hospital discharge, the recipient area was completely covered by skin flaps from the thighs.

appear some days after the initial event, at the end of the first week in most cases (Fig. 1). Highly unproportional wound pain as compared with the mild local changes, as well as leukocytosis, should alert one to this condition ${ }^{10}$. Gaseous infiltration of the soft tissues, detected by imaging methods or crepitus, is a classic sign, although its absence does not exclude necrotizing fasciitis ${ }^{7}$. Septic shock and multiorgan failure are common features and require prompt institution of fluid resuscitation and cardiorespiratory care ${ }^{8,9}$. Surgical exploration is the most important therapeutic and diagnostic tool, as it may demonstrate the lack of adherence between the necrotic fascia and its muscle ${ }^{7}$, another pathologic characteristic of necrotizing fasciitis ${ }^{9}$. In our case, the diagnosis made only on the $8^{\text {th }}$ postoperative day when crepitus, septic shock, and advanced soft tissue necrosis occurred. This delay is partially explained by the fact that necrotizing fasciitis is a rare infection after cardiac surgery, which in turn lowers our threshold of clinical suspicion.

Chest X-Ray, magnetic resonance imaging, computerized tomography, and ultrasound are additional examinations for eliciting the diagnoses ${ }^{9}$. Computerized tomography and ultrasound, as well as conventional X-ray, may evidence the presence of gas in affected tissues, mainly in diabetics ${ }^{11,7}$. Computerized tomography and magnetic resonance imaging are u seful for delimitation of the extent and spreading of the necrotizing infection ${ }^{12}$. In our reported ca- se, the suggested diagnosis of mediastinitis from computerized tomography scan could potentially have induced a more aggressive exploratory thoracotomy, reopening the sternum and extending the infection into the deep mediastinal structures. It is important to bear in mind that anterior and superior mediastinal liquid collections are commons and, in most cases are meaningless, in the first postoperative days of cardiac procedures. Clearly, with simultaneous evidence of septic shock and soft tissue infection, this differential diagnosis may become difficult, if not impossible.

Once the minor wound inflammatory changes have been excluded, the differential diagnosis should focus on necrotizing versus nonnecrotizing soft tissue infections. Increased leukocyte count, elevated BUN and low sodium concentration in the blood are all suggestive of necrotizing infection, and leukocytosis is of prognostic value, a predictive variable for higher mortality rates ${ }^{2}$. It should be remembered that pyoderma gangrenosum, a rare cutaneous disorder that affects predisposed individuals, that responds to surgical debridement and broad spectrum antibiotics, is less than ideal, ${ }^{13}$. In such cases, the basis for successful treatment are large doses of corticosteroids, of course, associated with aggressive surgical debridement and antibiotics. Myonecrosis or gas gangrene, caused by Clostridium perfringens, should also be kept in mind ${ }^{5}$.

The initial antibiotic choice should assure broadspectrum treatment including Gram-positives and Gram-negatives aerobes as well as anaerobes. An association of penicillin or cephalosporin with aminoglycosides plus clindamycin or metronidazole targeting anaerobes should be promptly started. Later on, according to the cultures and tests obtained from tissues and local discharge, the definitive antibiotic regimen will be instituted ${ }^{7}$. Third generation cephalosporins like ceftriaxone (Gram-negatives), cefazolin (aerobic Gram-positive coccus - Streptococcus), and vancomycin (Gram-positive coccus - Staphylococcus) is a reasonable association. It is important to emphasize that high doses of penicillin are still strongly recommended and are the best choice for treating necrotizing fasciitis due to Streptococcus pyogenes.

Our reported patient had to be maintained on an anticoagulation regimen with intravenous heparin due to the fact that a mechanical prosthetic valve was implanted along with the ascending aortic tubular graft. Heparin was suspended 4 hours before the surgical debridements and then restarted afterwards. Oral Coumadin anticoagulation was fully achieved only after definitive skin flap fixation and repair.

Hyperbaric oxygenation has been suggested as a complimentary method of treatment together with all the previous mentioned issues. It is based on the fact that once tissue oxygen tension is kept high, the end result would be increased leukocyte phagocytes in the edges of the wound. Although the Undersea and Hyperbaric Medical Society has strongly suggested the usefulness of the method, many studies have questioned the efficacy of this treatment in decreasing morbidity and mortality in necrotizing fasciitis ${ }^{9,15}$.

In spite of early and aggressive treatment, necrotizing 
fasciitis still carries high mortality rates. Quite different from other soft tissue infections, which can heal after simple incision and drainage, necrotizing fasciitis requires radical surgical debridement without delay, this last factor being res- ponsible for increments of morbidity and mortality. Nonthoracic necrotizing fasciitis mortality ranges from 15 to $50 \%$, and a thoracic location has the highest rate of $89 \%$. Elderly patients are at higher risk too ${ }^{5}$.

\section{References}

1. Lalwani AK, Kaplan MJ. Mediastinal and thoracic complications of necrotizing fasciitis of the head and neck. Head Neck 1991; 13: 531-9.

2. Wall DB, de Virgilio C, Black S. Objective criteria may assist in distinguishing necrotizing fasciitis from non-necrotizing soft tissue infection. Am J Surg 2000; 179: 17-21.

3. McArdle P, Gallen I. Necrotizing fasciitis in diabetics. The Lancet 1996; 348: 552

4. Urschel JD, Takita H, Antkowiak JG. Necrotizing soft tissue infections of the chest wall: current review. Ann Thorac Surg 1997; 64: 276-9.

5. Samsygin SA, Dolgina EN, Lukianov SV, Rudakov SS. Immunologic disorders in children with developmental thoracic defects. Khirurgiia (Mosk) 1990; 8: 85-9.
6. Green RJ, Dafoe DC, Raffin TA. Necrotizing fasciitis. Chest, 1996, 110 : 219-29.

7. File TM Jr, Tan JS. Treatment of skin and soft- tissue infections. Am J Surg 1995 169(5A-suppl): 27s-33s

8. Rahmouni A, Chosidow O, Mathieu D, et al. MR imaging in acute infectious cellulitis. Radiology 1994; 192: 493-6.

9. Rand RP, Olerud JE, Verrier ED. Pyoderma gangrenosum after coronary artery bypass grafting. Ann Thorac Surg 1993; 55:1016-8.

10. Shupak A, Shoshani O, Goldenberg I, et al. Necrotizing fasciitis: an indication for hyperbaric oxigenation therapy? Surgery 1995; 118: 873-8. 